\title{
Elderly-Onset Generalized Pustular Psoriasis without a Previous History of Psoriasis Vulgaris
}

\author{
Takayuki Aizu $^{a}$ Akinobu Matsui ${ }^{a} \quad$ Noriko Takiyoshi ${ }^{a}$ Eijiro Akasaka ${ }^{a}$ \\ Takahide Kaneko $^{a}$ Hajime Nakano ${ }^{a}$ Kazumitsu Sugiura ${ }^{b}$ \\ Masashi Akiyama ${ }^{\mathrm{b}}$ Daisuke Sawamura ${ }^{\mathrm{a}}$ \\ ${ }^{a}$ Department of Dermatology, Hirosaki University Graduate School of Medicine, Hirosaki, \\ and ${ }^{b}$ Department of Dermatology, Nagoya University Graduate School of Medicine, \\ Nagoya, Japan
}

\section{Key Words}

IL36RN · CARD14 · Pustulosis · Aging

\begin{abstract}
Generalized pustular psoriasis (GPP) is characterized by sudden fever and extensive erythema with pustules and occurs in patients with or without preceding psoriasis vulgaris. We report an 83-year-old man showing irregularly shaped erythema with pustules on the trunk and extremities. He initially had no fever and came to our clinic a few days after the onset of the skin lesions because of high fever and general malaise. We found an extension and new development of erythema and pustules on the whole body. The patient also manifested night delirium. Histological examination revealed neutrophil infiltration into the upper epidermis, which formed a spongiform pustule of Kogoj. Pustular fluid cultures were negative for bacteria. We diagnosed GPP without preceding psoriasis vulgaris. Mutation analysis revealed no significant mutations in IL36RN and CARD14. Previous reports indicated that onset of GPP at the age of 83 years is definitely rare. In older individuals, general disease characteristics include an atypical clinical course, an especially slow appearance and cure, and mental disorder. Our case also revealed such characteristics. Thus, it is necessary to be aware of the clinical course and mental problems in elderly patients with GPP.


Aizu et al.: Elderly-Onset Generalized Pustular Psoriasis without a Previous History of Psoriasis Vulgaris

\section{Introduction}

Generalized pustular psoriasis (GPP) is a rare severe psoriasis characterized by the presence of variable numbers of sterile pustules appearing in erythematous and scaly lesions. It can be lethal without proper treatment, especially in the elderly population. Mutations in IL36RN have been identified in familial GPP and in sporadic cases of GPP [1,2]. Cases with IL36RN mutations have been delineated as psoriasis with a deficiency of IL36RN (DITRA) [1]. Recently, the gene CARD14 has also been found to be responsible for GPP [3]. Herein, we report our recent experience with an 83-year-old man with GPP without a previous history of psoriasis vulgaris in whom we examined mutations in IL36RN and CARD14.

\section{Case Presentation}

An 83-year-old man with a 7-day history of erythematous eruption on the trunk and extremities was referred to our clinic. His history showed bronchial asthma, hypertension, atrial fibrillation, and postoperative colon carcinoma. He had never been previously diagnosed with psoriasis vulgaris. Physical examination revealed irregularly shaped erythema with scales and small pustules on the trunk and extremities (fig. 1). He had no fever. The blood test revealed a white blood cell count of $12,530 / \mu \mathrm{l}$ (segment $87.8 \%$, lymphocytes $6.7 \%$, monocytes $4.9 \%$, eosinophils $0.5 \%$, and basophils $0.1 \%$ ) and a C-reactive protein level of $2.2 \mathrm{mg} / \mathrm{dl}$.

We suspected pustular psoriasis and took a skin biopsy from an abdominal lesion. Since the patient had no fever, we started to treat him with oral antihistamine and topical steroid.

However, after 4 days, the patient came to our clinic because of high fever and general malaise. We found an extension and new development of erythema and pustules on the whole body (fig. 2). Coalescence of pustules resulted in lakes of pus. The blood test showed a white blood cell count of $18.920 / \mu \mathrm{l}$ (segment $88.4 \%$, lymphocytes $65.0 \%$, monocytes $6.3 \%$, eosinophils $0.2 \%$, and basophils $0.1 \%$ ) and a C-reactive protein level of $20.171 \mathrm{mg} / \mathrm{dl}$.

The patient was immediately admitted to our hospital ward. Although the results of pustular fluid culture and histopathology were not yet submitted, we started $60 \mathrm{mg} /$ day of oral etretinate, and pustule formation decreased (fig. 3). However, erythema continued to expand, and consequently the lesions became erythrodermic. In addition, a decrease in serum albumin caused general edema, and night delirium appeared. Afterwards, erythema gradually decreased, and we tapered the dose of etretinate. Administration of diuretic furosemide was effective for general edema. The delirium gradually disappeared.

Later, histological examination revealed neutrophil infiltration into the upper epidermis, which formed a spongiform pustule of Kogoj (fig. 4). Direct immunofluorescence showed no significant deposits of immunoglobulins. Pustular fluid cultures were negative for bacteria. We performed mutation analysis, which detected no significant mutations in IL36RN and CARD14.

\section{Discussion}

GPP is characterized by sudden fever and extensive erythema with pustules and occurs in patients with or without psoriasis vulgaris. Since this case showed pustule formation on the whole body and no history of psoriasis vulgaris, the differential diagnosis initially included GPP, subcorneal pustular dermatosis, acute generalized exanthematous pustulosis, 
Aizu et al.: Elderly-Onset Generalized Pustular Psoriasis without a Previous History of Psoriasis Vulgaris

and intercellular immunoglobulin IgA dermatosis. However, clinical, histological, and immunofluorescence findings, in conjunction with a lack of recent administration of causative drugs, resulted in a final diagnosis of GPP.

Recently, mutations in IL36RN and CARD14 have been found to be responsible for GPP $[1,2]$. The IL36RN protein is primarily expressed in the skin and is an antagonist of three cytokines that belong to the interleukin-1 family: interleukin-36 $\alpha$, interleukin-36 $\beta$, and interleukin-36 $\gamma$, which are also known as interleukin-1F6, interleukin-1F8, and interleukin$1 \mathrm{~F}$, respectively [4]. These cytokines activate several proinflammatory signaling pathways, such as the nuclear factor- $\mathrm{\kappa B}$ and mitogen-activated protein kinase pathways [4]. Therefore, loss of function of IL36RN may cause GPP. Tominaga et al. [5] reported that a 78-year-old woman with GPP and a previous history of psoriasis vulgaris had causative mutations in the $I L 36 R N$ gene. Since it was possible that our patient might have had mutations in IL36RN or CARD14, we performed mutation analysis. However, we were unable to find any of the known mutations.

An interesting point of this case is that the onset of GPP occurred at an older age. The age of onset in Japanese patients recently reported in reliable English-language papers was 2-78 years in 31 cases [6], 36-71 years in 3 cases [7], 16-74 years in 14 cases [8], and 21-64 years in 3 cases [9]. In addition, the age of onset in a recent report from Malaysia was 21-81 years in 102 cases [10]. These reports suggest that the age of onset in the present case (at 83 years) is definitely rare. In older individuals, general disease characteristics include an atypical clinical course, an especially slow appearance, a slow cure, and mental disorder. In our case, we were initially unable to recognize the severity of the disease because of the lack of fever and a low C-reactive protein level. Three days after his first visit, however, sudden fever and extension of the skin lesions were detected, and he reported night delirium. This illustrates the need to be aware of the clinical course and mental problems in elderly patients with GPP.

\section{Statement of Ethics}

The authors state that the patient gave his informed consent, and the informed consent was in accordance with the guidelines approved by Hirosaki University Graduate School of Medicine.

\section{Disclosure Statement}

The authors declare that there are no conflicts of interest.

\section{References}

1 Marrakchi S, Guigue P, Renshaw BR, Puel A, Pei XY, Fraitag S, Zribi J, Bal E, Cluzeau C, Chrabieh M, Towne JE, Douangpanya J, Pons C, Mansour S, Serre V, Makni H, Mahfoudh N, Fakhfakh F, Bodemer C, Feingold J, HadjRabia S, Favre M, Genin E, Sahbatou M, Munnich A, Casanova JL, Sims JE, Turki H, Bachelez H, Smahi A: Interleukin-36-receptor antagonist deficiency and generalized pustular psoriasis. N Engl J Med 2011;365:620-628.

-2 Onoufriadis A, Simpson MA, Pink AE, Di Meglio P, Smith CH, Pullabhatla V, Knight J, Spain SL, Nestle FO, Burden AD, Capon F, Trembath RC, Barker JN: Mutations in IL36RN/IL1F5 are associated with the severe episodic inflammatory skin disease known as generalized pustular psoriasis. Am J Hum Genet 2011;89:432437. 
Aizu et al.: Elderly-Onset Generalized Pustular Psoriasis without a Previous History of Psoriasis Vulgaris

3 Jordan CT, Cao L, Roberson ED, Pierson KC, Yang CF, Joyce CE, Ryan C, Duan S, Helms CA, Liu Y, Chen Y, McBride AA, Hwu WL, Wu JY, Chen YT, Menter A, Goldbach-Mansky R, Lowes MA, Bowcock AM: PSORS2 is due to mutations in CARD14. Am J Hum Genet 2012;90:784-795.

-4 Dinarello C, Arend W, Sims J, Smith D, Blumberg H, O'Neill L, Goldbach-Mansky R, Pizarro T, Hoffman H, Bufler P, Nold M, Ghezzi P, Mantovani A, Garlanda C, Boraschi D, Rubartelli A, Netea M, van der Meer J, Joosten L, Mandrup-Poulsen T, Donath M, Lewis E, Pfeilschifter J, Martin M, Kracht M, Muehl H, Novick D, Lukic M, Conti B, Solinger A, Kelk P, van de Veerdonk F, Gabel C: IL-1 family nomenclature. Nat Immunol 2010;11:973.

-5 Tominaga C, Yamamoto M, Imai Y, Yamanishi K: A case of old age-onset generalized pustular psoriasis with a deficiency of IL-36RN (DITRA) treated by granulocyte and monocyte apheresis. Case Rep Dermatol 2015;7:29-35.

-6 Sugiura K, Takemoto A, Yamaguchi M, Takahashi H, Shoda Y, Mitsuma T, Tsuda K, Nishida E, Togawa Y, Nakajima K, Sakakibara A, Kawachi S, Shimizu M, Ito Y, Takeichi T, Kono M, Ogawa Y, Muro Y, IshidaYamamoto A, Sano S, Matsue H, Morita A, Mizutani H, Iizuka H, Muto M, Akiyama M: The majority of generalized pustular psoriasis without psoriasis vulgaris is caused by deficiency of interleukin-36 receptor antagonist. J Invest Dermatol 2013;133:2514-2521.

-7 Suzuki A, Haruna K, Mizuno Y, Kuwae Y, Ono Y, Okumura K, Negi O, Kon Y,Takeuchi K, Takamori K, Ikeda S, Suga Y: Successful treatment of three cases of generalized pustular psoriasis with granulocyte and monocyte adsorption apheresis. Ther Apher Dial 2012;16:445-448.

-8 Farooq M, Nakai H, Fujimoto A, Fujikawa H, Matsuyama A, Kariya N, Aizawa A, Fujiwara H, Ito M, Shimomura Y: Mutation analysis of the IL36RN gene in 14 Japanese patients with generalized pustular psoriasis. Hum Mutat 2013;34:176-183.

-9 Fujisawa T, Murase K, Okumura Y, Kanoh H, Doi T, Yoshida S, Ogura S, Seishima M: Generalized pustular psoriasis successfully treated with granulocyte and monocyte adsorption apheresis. Ther Apher Dial 2011;15:374-378.

10 Choon SE, Lai NM, Mohammad NA, Nanu NM, Tey KE, Chew SF: Clinical profile, morbidity, and outcome of adult-onset generalized pustular psoriasis: analysis of 102 cases seen in a tertiary hospital in Johor, Malaysia. Int J Dermatol 2015;53:676-684. 


\section{Case Reports in Dermatology}

Aizu et al.: Elderly-Onset Generalized Pustular Psoriasis without a Previous History of Psoriasis Vulgaris
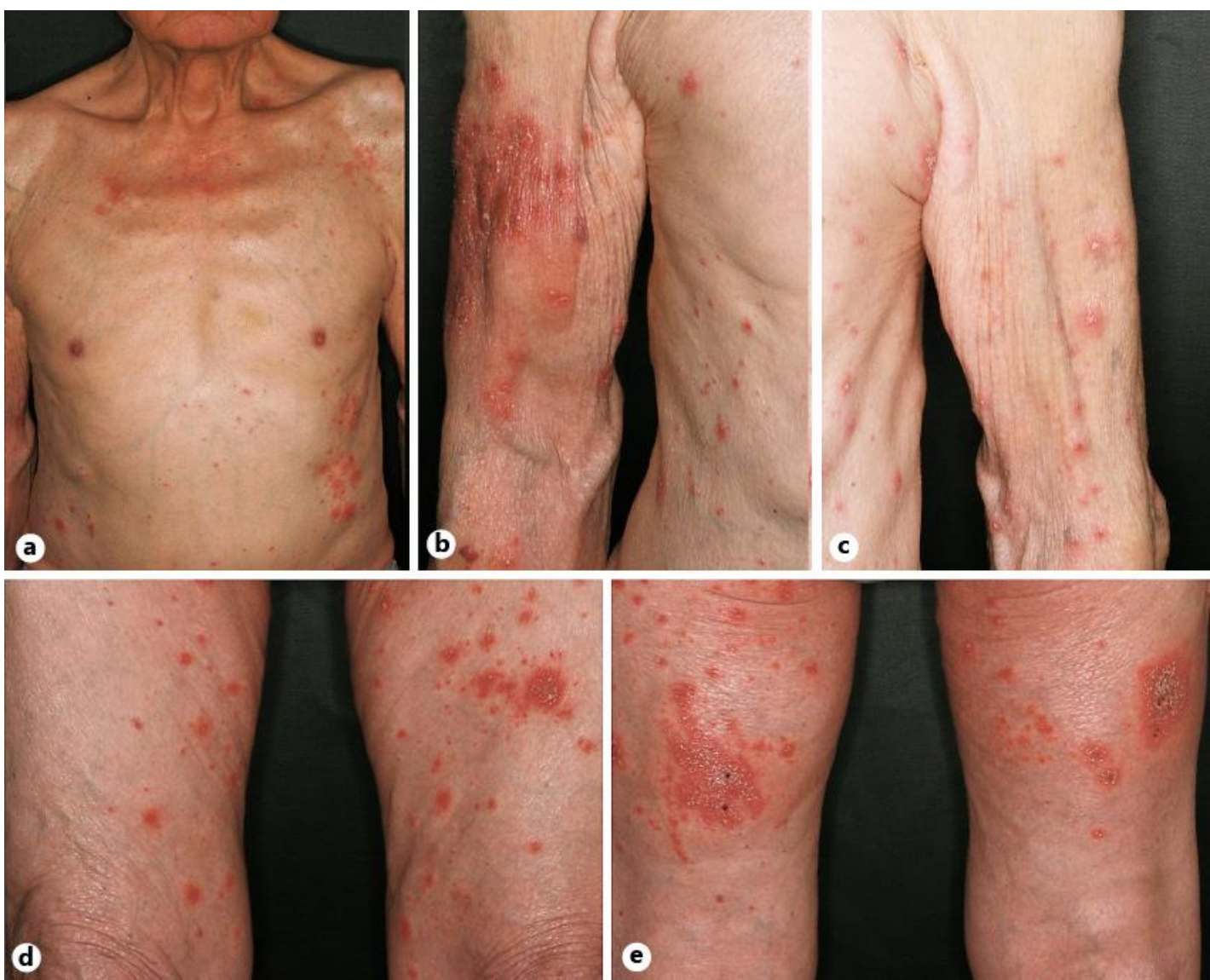

Fig. 1. Clinical findings at his first visit. Irregularly shaped erythema with scales and small pustules on the trunk and extremities. The patient had no fever. a Trunk. b, c Upper extremities. d, e Thighs. 


\section{Case Reports in Dermatology}
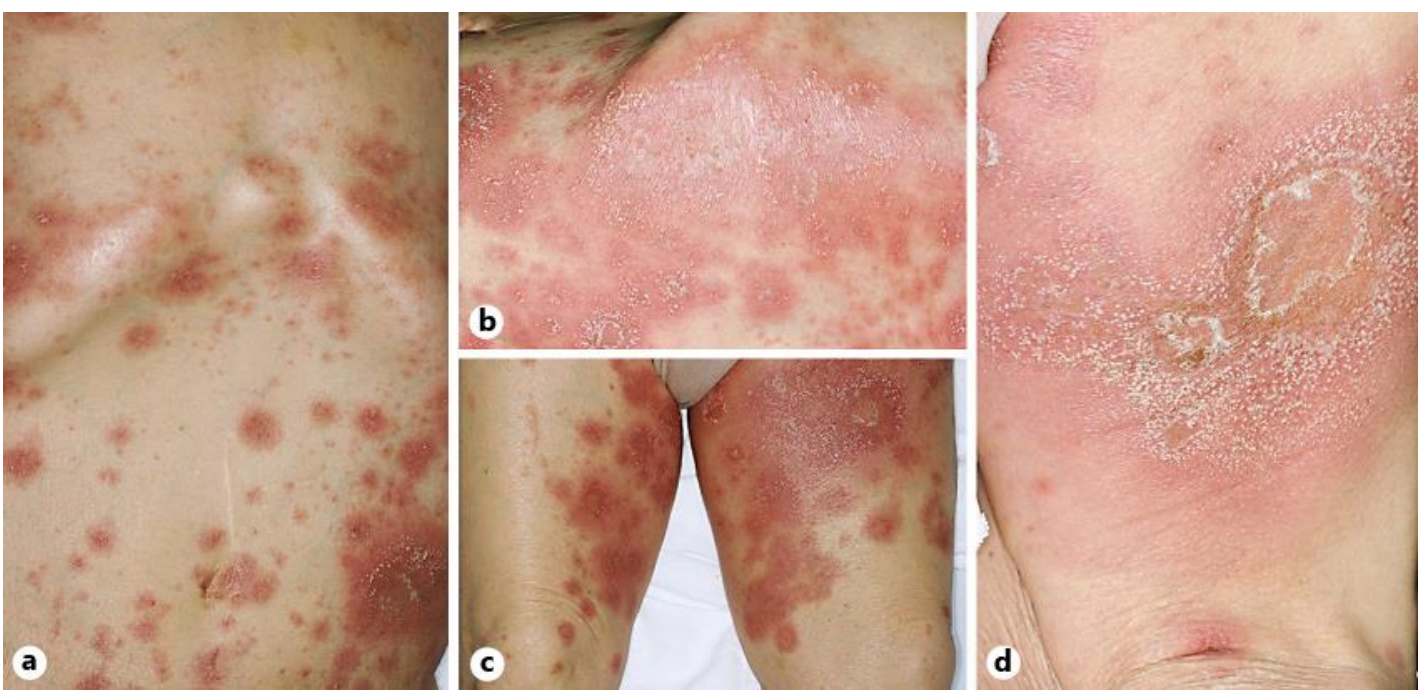

Fig. 2. Clinical findings at his second visit. The patient came to our clinic because of high fever and general malaise. We found an extension and new development of erythema and pustules on the whole body. a Abdomen. b Buttock. c, d Thighs.

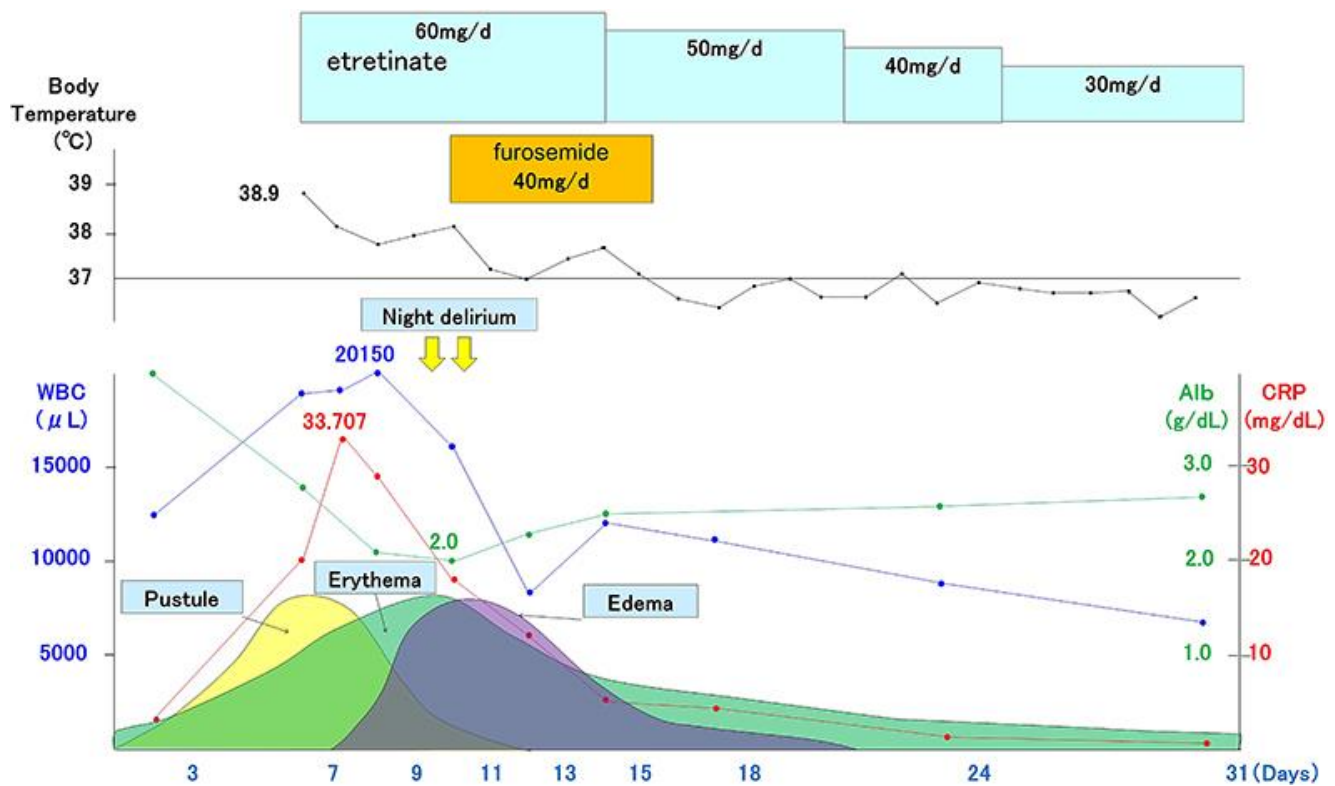

Fig. 3. Clinical course and treatments. 
Aizu et al.: Elderly-Onset Generalized Pustular Psoriasis without a Previous History of Psoriasis Vulgaris

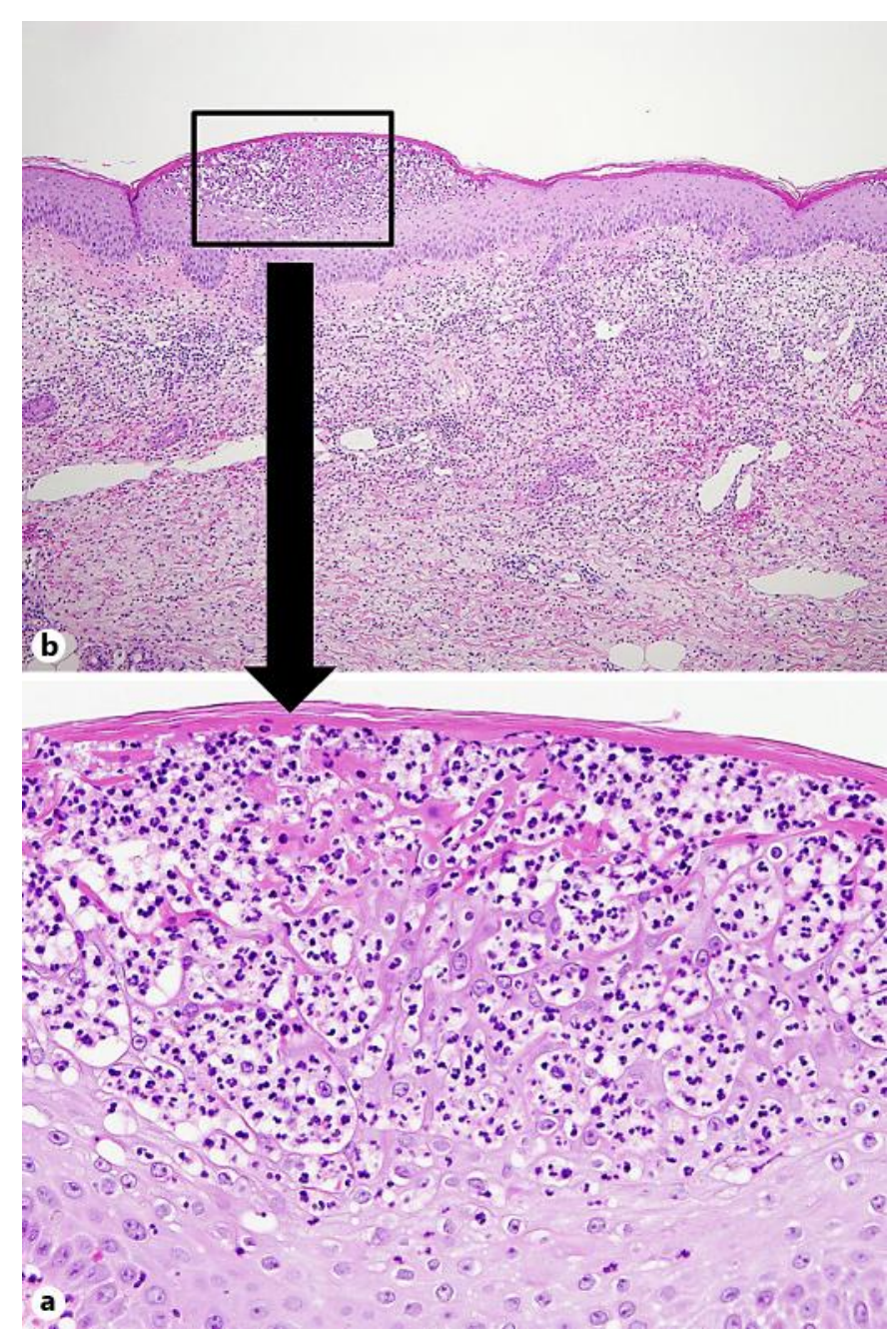

Fig. 4. a, b Histopathological findings. Neutrophil infiltration into the upper epidermis, which formed a spongiform pustule of Kogoj. $\mathbf{b}$ An enlarged photo of a part of $\mathbf{a} . \mathbf{a} \times 40 . \mathbf{b} \times 200$. 